\title{
Distance to SN 1987A and the LMC
}

\author{
Nino Panagia ${ }^{1}$ \\ STScI, 3700 San Martin Drive, Baltimore, MD 21218, USA
}

\begin{abstract}
Using the new reductions of the IUE light curves by Sonneborn et al. (1997) and an extensive set of HST images of SN 1987A we have repeated and improved Panagia et al. (1991) analysis to obtain a better determination of the distance to the supernova. In this way we have derived an absolute size of the ring $R_{a b s}=(6.23 \pm 0.08) \times 10^{17} \mathrm{~cm}$ and an angular size $R^{\prime \prime}=808 \pm 17$ mas, which give a distance to the supernova $d(S N 1987 A)=51.4 \pm 1.2 \mathrm{kpc}$ and a distance modulus $m-$ $M(S N 1987 A)=18.55 \pm 0.05$. Allowing for a displacement of SN 1987A position relative to the $\mathrm{LMC}$ center, the distance to the barycenter of the Large Magellanic Cloud is also estimated to be $d(L M C)=52.0 \pm 1.3 \mathrm{kpc}$, which corresponds to a distance modulus of $m-M(L M C)=18.58 \pm 0.05$.
\end{abstract}

\section{Introduction}

In 1991 Panagia et al. estimated the distance to SN 1987A $(51.2 \pm 3.1 \mathrm{kpc})$ from a comparison of the angular size of the inner circumstellar ring as measured with the HST-FOC in August 1990 (Jakobsen et al. 1991), with the ring absolute size as determined from the peaks of its UV emission line light curves.

Recently, Gould re-examined the problem adopting an infinitely narrow ring geometry and retaining the Panagia et al. (1991) assumption of an exponential law for the line emissivity (Gould 1995; Gould \& Uza 1998). Thus, using the same data as in Panagia et al. (1991) and Sonneborn et al. (1997), respectively, but including only the NIV] and NIII] light curves, and adopting the average [OIII] ring size as measured by Plait et al. (1995) over the period August 1990 May 1993, Gould concluded that the distance to SN 1987A is less than $47 \mathrm{kpc}$.

The new reductions of the IUE spectra, done by Sonneborn et al. (1997), have produced more accurate and reliable light curves. Therefore, we have decided to repeat Panagia et al. (1991) analysis using the new data set and including a more accurate and realistic estimate of the ring angular size obtained from the study of an extensive set of HST images of SN 1987A. Here we present a brief outline of our analysis and the main results of our study. A complete account of this work will be presented in a forthcoming paper (Panagia et al. 1999).

\footnotetext{
${ }^{1}$ On assignment from the Astrophysics Division, Space Science Department of ESA.
} 


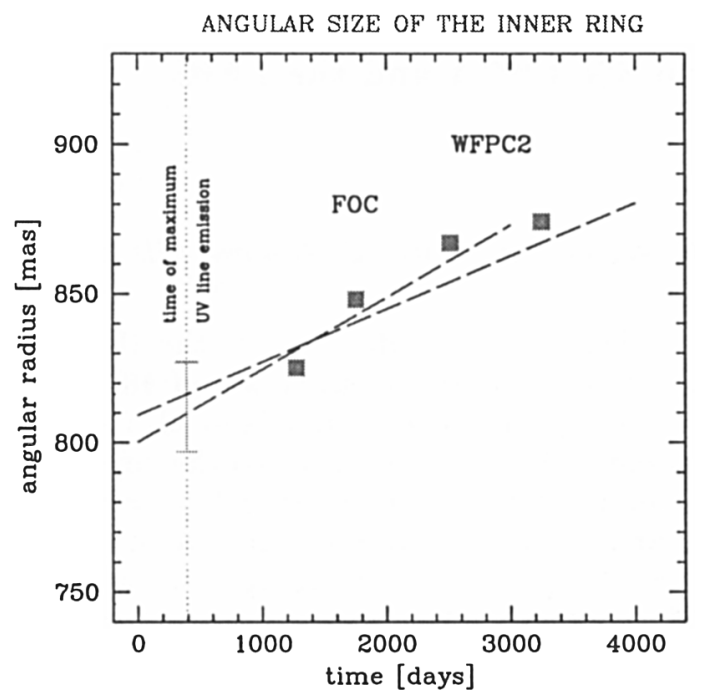

Figure 1. The average angular radius of the ring as measured in $F O C$ and $W F P C 2$ images taken with an [OIII] filter. Two fits to the observed points and the range of possible values of the radius at the time of the UV maximum are shown.

\section{The Angular Size of the Ring}

The inner circumstellar ring is clearly extended with a HPW of about $1 / 7$ its radius (e.g., Jakobsen et al. 1991; Panagia et al. 1999). The finite width of the ring makes the definition of an average size a very delicate one, which, if done improperly, may introduce errors as large as, say, $7 \%$ or higher.

The analysis of both FOC and WFPC2 images have revealed that the ring angular size, as estimated from [OIII] images, appears to increase with time (Panagia et al. 1999; see Fig. 1) while the one derived from $\mathrm{H} \beta$ and [NII] images remains constant in time and virtually coincides with the size measured in the earliest [OIII] image. This is the effect of both cooling and recombination of the $\mathrm{O}^{++}$ion, that cause the [OIII] $5007 \AA$ line intensity to decline more quickly at the inner edge of the ring where the density is higher. Therefore, the correct value of the ring angular size to compare with the absolute size determined from the UV lines is an extrapolation of the observed sizes, as measured in the [OIII] band, back to the epoch of maximum UV line emission (approximately early April 1988; $c f$. Fig. 1). In this way we obtain:

$$
R^{\prime \prime}=808 \pm 17 \operatorname{mas}
$$




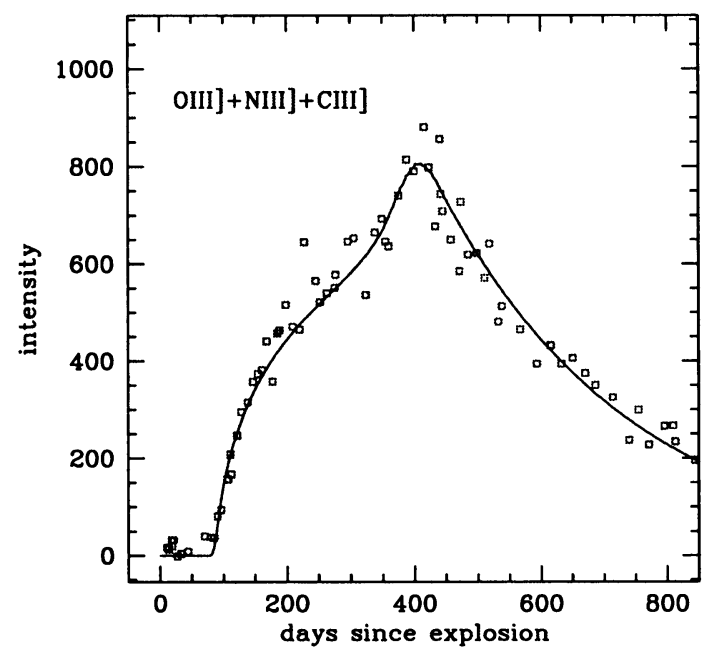

Figure 2. The sum of the observed intensities (in units of $10^{-15} \mathrm{erg}$ $\mathrm{cm}^{-2} \mathrm{~s}^{-1}$ ) of the OIII] $\left.1666 \AA, \mathrm{NIII}\right] 1750 \AA$, and CIII] $1909 \AA$ lines compared to the best-fit model light curve.

\section{The Absolute Size of the Ring}

It has been shown (Panagia et al. 1991; Dwek \& Felten 1992; Gould 1995) that under the assumption of an infinitely narrow width the absolute radius of the ring is simply related to the onset time of the UV line emission $t_{\mathrm{o}}$, and the time of maximum UV line emission $t_{\max }$ as

$$
R=c\left(t_{\mathrm{o}}+t_{\max }\right) / 2
$$

Since the angular size is well measured only for $\mathrm{O}^{++}$, we have limited our analysis to the UV light curves of twice ionized ions, namely OIII, NIII and CIII, and we have compared them to theoretical light curves computed under the following assumptions: (1) The ring is circular with a width of $14 \%$ the ring radius; (2) The intrinsic emission of each ion decays exponentially with time; (3) The model free parameters are: radius and inclination angle of the ring, and specific emissivity at time $t=0$ and decay time of each line.

We computed best fits to both the individual light curves of the OIII], NIII], and CIII] lines, and the composite light curve, sum of the OIII], NIII] and CIII] line intensities (Fig. 2). The individual radius determinations fall in the range 6.0 to $6.4 \times 10^{17} \mathrm{~cm}$, with uncertainties of about $4 \%$ for $\left.\mathrm{OIII}\right]$ and $\left.\mathrm{CIII}\right]$, and slightly above $1 \%$ for NIII], resulting in an average value:

$$
R=(6.23 \pm 0.08) \times 10^{17} \mathrm{~cm}
$$




\section{Distance Determinations}

SN 1987A - Comparing the absolute and the angular sizes of the circumstellar ring, we derive a distance to $\mathrm{SN} 1987 \mathrm{~A}$

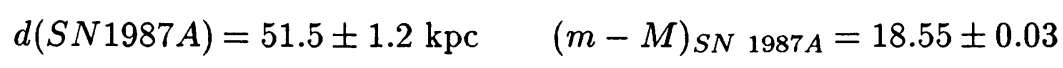

This value is very close to the original determination by Panagia et al. (1991) but is considerably more accurate. In the literature one finds determinations of SN 1987A distance which are all based on the analysis of UV line light curves and $H S T$ imaging but provide quite discrepant values, for example distance moduli ranging from 18.37 to 18.67 (Gould \& Uza 1998; Sonneborn et al. 1997; Lundqvist \& Sonneborn 1997; Panagia et al. 1999), all based on the same set of data. A close scrutiny of these papers reveals that all discrepancies are due to somewhat arbitrary differences in the angular size adopted and/or in the selection of UV emission lines light curves to determine the absolute size.

LMC - Allowing for the difference of the position of SN 1987A relative to the LMC barycenter (McCall 1993, 1994; also Crotts, Kunkel \& Heathcote, 1995; Crotts 1998, this Conference; Panagia et al 1999) the best estimate of the distance to the center of mass of the LMC is found to be

$$
d(L M C)=52.1 \pm 1.3 \mathrm{kpc} \quad(m-M)_{L M C}=18.58 \pm 0.03
$$

The error includes the uncertainties on the SN $1987 \mathrm{~A}$ distance $( \pm 1.2 \mathrm{kpc})$ as well as those on the depth toward SN $1987 \mathrm{~A}( \pm 0.2 \mathrm{kpc})$ and the relative position of the LMC barycenter $( \pm 0.3 \mathrm{kpc})$.

Our determination of the LMC distance modulus $(18.58 \pm 0.05)$ is in excellent agreement with recent determinations obtained via Hipparcos parallax calibration of both galactic Cepheids (Feast \& Catchpole 1997: $(m-M)=$ $18.70 \pm 0.10)$ and local subdwarfs (Gratton et al. 1997: $(m-M)=18.60 \pm 0.07)$. A weighted average of the three determinations gives

$$
<(m-M)_{L M C}>=18.60 \pm 0.04
$$

It is apparent that the LMC distance modulus is around 18.60 and that values $<18.48$ and $>18.72$ are to be excluded with high confidence.

\section{References}

Crotts, A.P.S., Kunkel, W.E., \& Heathcote, S.R. 1995, ApJ, 438, 724.

Dwek, E., \& Felten, J.E. 1992, ApJ, 387, 551.

Feast, M.W., \& Catchpole, R.M. 1997, MNRAS, 286, L1.

Gould, A. 1995, ApJ, 452, 189.

Gould, A., \& Uza, O. 1998, ApJ, 494, 118.

Grattom, R., et al. 1997, ApJ, 491, 749.

Jakobsen, P., et al. 1991, ApJ, 369, L63. 
Lundqvist, P., \& Sonneborn, G. 1998, preprint.

McCall, M.L. 1993, ApJ, 417, L75; and erratum 1994, ApJ, 434, L37.

Panagia, N., Gilmozzi, R., Macchetto, F., Adorf, H.-M., \& Kirshner, R.P. 1991, ApJ, 380, L23.; and erratum 1992, ApJ, 386, L31.

Panagia, N., Gilmozzi, Kirshner, R.P., Pun, C.S.J., \& Sonneborn, G. 1999, ApJ, to be submitted.

Plait, P.C., Lundqvist, P., Chevalier, R.A., \& Kirshner, R.P. 1995, ApJ, 439, 730.

Sonneborn, G., et al. 1997, ApJ, 477, 848.

\section{Discussion}

Arlin Crotts: You should subtract at least $400 \mathrm{pc}$, and better $500 \mathrm{pc}$, rather than $100 \mathrm{pc}$ from the distance to $\mathrm{LMC}$ as determined by echo distance of SN 1987A behind the plane of LMC.

Doug Welch: 1. Can you comment on the degree to which ring clumpiness affects the distance derived?

2. Why do you favor the particular extrapolation to 0 days which you chose for the angular diameter?

Panagia: 1. Clumpiness does not affect the shape of the UV line light curves with the exception of two mysterious spikes at $\sim 200^{d}$ and $400^{d}$, which indicate the existence of two very small ( $\leq 20$ light-days) and very dense $\left(n_{e}>10^{5}\right.$ $\mathrm{cm}^{-3}$ ) clumps. All other knots are much less extreme and their effects average out completely.

2. My extrapolation of the [OIII] ring size to early times is bracketed between the smallest size measured (Aug 1990, 825 mas) in [OIII] images, and the smallest size measured in any radiation (i.e., [OI] in April 1997, 790 mas).

George Sonneborn: What distance to SN 1987A do you find if you assume that the [OI] diameter (Sonneborn et al. 1998, ApJ 492, L139) corresponds to the angular size of the ring at UV line maximum? This is plausible because the brightest UV emission originated from the densest gas, which recombined faster, and now would be neutral.

Panagia: The distance corresponding to the [OI] diameter you measure is 52.6 $\mathrm{kpc}$ or, similarly, a distance modulus of 18.60 . These values are essentially my quoted $1 \sigma$ upper limit to the distance to SN $1987 \mathrm{~A}$. 

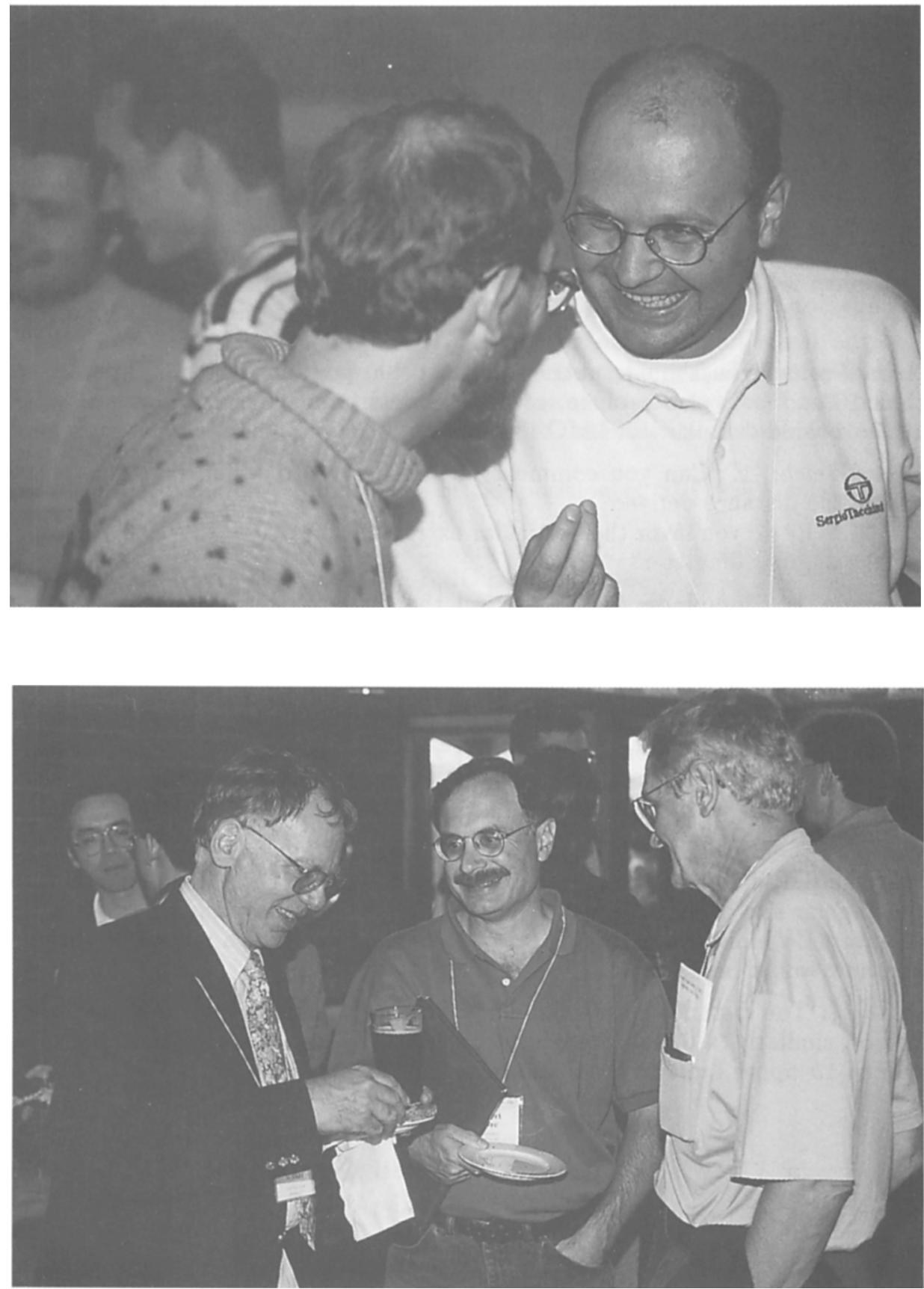

(Top) Miroslav Filipović enjoying a good story at the reception, as are (bottom) Frank Winkler, Robert Petre and Ted Stecher. 